\title{
A toddler PCV booster dose following 3 infancy priming doses increases circulating serotype-specific IGG levels but does not increase protection against carriage
}

\section{Dagan, Ron}

\section{8-05-11}

Dagan , R , Ben-Shimol , S , Simell , B , Greenberg , D , Porat , N , Käyhty , H \& Givon-Lavi , N 2018, ' A toddler PCV booster dose following 3 infancy priming doses increases circulating serotype-specific IGG levels but does not increase protection against carriage ' , Vaccine , vol. 36 , no. 20 , pp. 2774-2782 . https://doi.org/10.1016/j.vaccine.2018.04.007

http://hdl.handle.net/10138/302423

https://doi.org/10.1016/j.vaccine.2018.04.007

publishedVersion

Downloaded from Helda, University of Helsinki institutional repository.

This is an electronic reprint of the original article.

This reprint may differ from the original in pagination and typographic detail.

Please cite the original version. 


\title{
A toddler PCV booster dose following 3 infancy priming doses increases circulating serotype-specific IGG levels but does not increase protection against carriage
}

\author{
Ron Dagan $^{\mathrm{a}, *}$, Shalom Ben-Shimol ${ }^{\mathrm{a}, \mathrm{b}}$, Birgit Simell ${ }^{\mathrm{c}}$, David Greenberg ${ }^{\mathrm{a}, \mathrm{b}}$, Nurith Porat ${ }^{\mathrm{a}, \mathrm{b}}$, Helena Käyhty ${ }^{\mathrm{d}}$, \\ Noga Givon-Lavi a,b
}

${ }^{a}$ The Faculty of Health Sciences, Ben-Gurion University of the Negev, Beer-Sheva, Israel

${ }^{\mathrm{b}}$ The Pediatric Infectious Disease Unit, Soroka University Medical Center, Beer-Sheva, Israel

${ }^{\mathrm{C}}$ Institute for Molecular Medicine Finland (FIMM), University of Helsinki, Helsinki, Finland

${ }^{\mathrm{d}}$ Department of Vaccination and Immune Protection, National Institute for Health and Welfare (THL), Helsinki, Finland

\section{A R T I C L E I N F O}

\section{Article history:}

Received 7 January 2018

Received in revised form 30 March 2018

Accepted 3 April 2018

Available online 11 April 2018

\section{Keywords:}

Pneumococcal conjugate vaccines

PCV

Carriage

Serologic response

Booster

Vaccine schedule

Infants

Toddlers

\begin{abstract}
A B S T R A C T
Background: We compared PCV7 serological response and protection against carriage in infants receiving 3 doses $(2,4,6$ months; $3+0$ schedule) to those receiving a booster ( 12 months; $3+1)$.

Methods: A prospective, randomized controlled study, conducted between 2005 and 2008, before PCVs were implemented in Israel. Healthy infants were randomized $1: 1: 1$ to receive $3+1,3+0$ and $0+2$ (control group; 12, 18 months doses). Nasopharyngeal/oropharyngeal swabs were obtained at all visits. Serum serotype-specific IgG concentrations and opsonic activities (OPA) were measured at 2, 7, 13 and 19 months. This study was registered with Current Controlled Trials, Ltd. ISRCTN28445844.

Results: Overall, 544 infants were enrolled: $3+1(n=178), 3+0(n=178)$ and $0+2(n=188)$.

Post-priming ( 7 months), antibody concentrations were similar in both groups, except for serotype $18 \mathrm{C}$ (higher in 3+0). Post-booster (13, 19 months), ELISA and OPA levels were significantly higher in 3+1 than in $3+0$ group.

Nasopharyngeal/oropharyngeal cultures were positive for Streptococcus pneumoniae in 2673 (54.3\%) visits. Acquisition rates (vaccine and non-vaccine serotypes) were similar for $3+1$ and $3+0$ groups at $7-$ 30 months and for 0+2 group at 19-30 months.

Conclusions: PCV7 booster after 3 priming doses increased substantially IgG concentrations but did not further reduced vaccine-serotype nasopharyngeal acquisition, suggesting that protection from pneumococcal carriage does not depend primarily on serum IgG.
\end{abstract}

(c) 2018 Elsevier Ltd. All rights reserved.

\section{Introduction}

The 7-valent $\mathrm{CRM}_{197}$ pneumococcal conjugate vaccine (PCV7) was licensed in the US in the early 2000s for use as 3 infant doses and a booster in second year of life $(3+1$ schedule) [1]. Subsequently, many countries introduced pneumococcal conjugate vaccines (PCVs) into their national immunization program (NIP) $[2,3]$.

Abbreviations: 7VT+6A, pneumococcal serotypes included in PCV7 and serotype 6A; MCHCs, Mother-and-Child Health Centers; MOPA, multiplexed opsonophagocytosis assay; NIP, national immunization program; OPA, opsonic activities; PCVs, pneumococcal conjugate vaccines; $\mathrm{PCV} 7,7$-valent $\mathrm{CRM}_{197}$ pneumococcal conjugate vaccine; PCV13, 13-valent conjugate vaccine.

* Corresponding author at: The Pediatric Infectious Disease Unit, Soroka Univer-

sity Medical Center, Beer-Sheva, Israel.

E-mail address: rdagan@bgu.ac.il (R. Dagan).
However, considerations regarding the high cost of PCVs and the growing number of injections in infant vaccination regimens led many countries to choose different (reduced) schedules with no toddler dose $(3+0)$ and 2 infant doses with a toddler booster dose $(2+1)[4]$.

Schedules with a lower number of doses often elicit lower immune response compared to the original $3+1$ schedule [5-11]. Furthermore, a higher nasopharyngeal carriage rate with decreasing number of PCV doses in infants was observed in several studies $[6,12,13]$. However, the clinical consequences of the immunologic differences are not entirely clear and are subject to debate $[10,11]$. Systematic reviews support a $3+0$ schedule, which in various studies was typically used in infants at 6,10 , and 14 weeks or 2,4 , and 6 months schedules (the latter was often studied compared with a booster toddler dose as a $3+1$ schedule) $[10,14]$. 
Reduction in nasopharyngeal carriage of pneumococcal serotypes included in PCV7 and serotype 6A (7VT+6A) plays an important role in the success of immunization programs, by reducing transmission to susceptible subjects, resulting in indirect (herd) protection $[15,16]$. In the US, a near total elimination of 7VT+6A was observed in all ages in the PCV7 era [17]. Several studies have shown that a $3+0$ regimen was also associated with carriage reduction $[8,13,18-20]$. Furthermore, after the introduction of the $3+0$ PCV7 regimen to the NIP in Australia, indirect protection was demonstrated [21]. However, these reports do not enable comparison of the $3+0$ and $3+1$ regimens, and to determine whether the 3 +0 regimen is equivalent to the $3+1$ regimen in its ability to induce and maintain reduction in 7VT+6A pneumococcal serotypes in the community. Furthermore, a recent study from Australia suggested that after 13-valent conjugated vaccine (PCV13) introduction with the $3+0$ regimen, a lower herd protection than expected was observed, prompting a change to a $3+1$ regimen [22].

We conducted a prospective randomized controlled study prior to PCV7 implementation in Israel to examine the added value of the booster dose in protecting against carriage in toddlers who had received 3 infancy doses by comparing the effect of the $3+0$ and $3+1$ responses on acquisition and prevalence of pneumococcal carriage in infants and toddlers. We also included in this group of children those who were not vaccinated in the first year of life, but received a 2 -dose regimen at 12 and 18 months $(0+2)$, to assess the toddler-only schedule on carriage, as this schedule only protects directly against infections caused by vaccine-serotypes in toddlers and does not protect directly against infections in infants.

\section{Methods}

\subsection{Setting}

Two ethnic groups whose socioeconomic conditions and lifestyles differ inhabit Southern Israel (the Negev region): the Jewish and the Bedouin populations. The Bedouin population, formerly desert nomads, in transition to a western lifestyle, is characterized by overcrowding, lower levels of education, lower income and larger family size than the Jewish population [23]. Pneumococcal nasopharyngeal carriage is usually higher among Bedouin infants than among Jewish infants [24]. Approximately 95\% of all routine immunizations in Israel are given in public sector Mother-andChild Health Centers (MCHCs). In the present study, we selected 7 MCHCs, 4 serving the Jewish population and 3 serving the Bedouin population (yearly birth cohorts of $\sim 1400$ and $\sim 1700$, respectively).

PCV7 was licensed in Israel in 2006 and became publicly available in 2007 . However, in southern Israel, only $<10 \%$ and $<25 \%$ of children received the vaccine before 2008 and between 2008 and mid-2009, respectively. In July 2009, PCV7 was implemented in the Israeli NIP.

\subsection{Participants}

Healthy infants were enrolled at the age of 2 months $( \pm 3$ weeks). Exclusion criteria were: prematurity ( $<35$ weeks); fever $\left(>38^{\circ} \mathrm{C}\right)$; congenital abnormalities; metabolic disorders; coagulation disorders; use of immune-modifying drugs; allergy to $\geq 1$ vaccine constituents; hypotonic-hyporesponsiveness or persistent inconsolable crying after any prior vaccine; and HIV infection.

The study was approved by the Ethics Committees of the Soroka University Medical Center, Maccabi Health Services and the Ministry of Health and was registered with Current Controlled Trials, Ltd. ISRCTN28445844.

\subsection{Study design}

This open-label study was initiated in August 2005. Enrollment was conducted through March 2008, and the last follow-up visit was in March 2009, before the implementation of PCV7 in the Israeli NIP. We invited $\sim 3100$ eligible infants who belonged to the study MCHCs to take part in the study. The parents of 1141 infants were willing to consider participation in the study, and eventually 733 infants were enrolled [12]. After an informed consent was obtained, subjects were randomized $1: 1: 1: 1$ to receive 3 PCV7 doses at 2, 4, 6 months and a 4th toddler dose at 12 months, $(3+1 ; n=178) ; 3$ PCV7 doses at 2, 4, 6 months with no additional toddler doses $(3+0 ; n=178)$; and no dose during infancy but 2 doses in the second year at 12, 18 months (control group, 0 $+2 ; \mathrm{n}=188$ ) (Fig. 1) [12]. The third group, originally studied as a "catch-up" schedule for the purpose of PCV7 national immunization plan introduction [12] was chosen as a control group for both the $3+1$ and $3+0$ groups, since they did not receive any PCV7 dose during their first year of life. An additional 189 subjects were enrolled to a 2+1 arm (presented in a previous article) [12].

The study vaccine was Prevenar ${ }^{\mathrm{TM}}$ (Wyeth Vaccines; Collegeville, PA, USA) containing 7 serotypes (4, 6B, 9V, 14, 18C, 19F and 23F) individually conjugated to $\mathrm{CRM}_{197}$; lots used were no. A94431D, B08636C, 808672K and C659732), All infants received concomitant vaccines.

Blood (2-4 mL) was drawn at ages 2, 7, 13 and 19 months, transferred refrigerated within $8 \mathrm{~h}$ to the laboratory, separated within $24 \mathrm{~h}$ and kept at $-70{ }^{\circ} \mathrm{C}$ until processed. In group $0+2$, subjects were randomized (1:1) to give blood samples at 2 months or at 7 months of age, but all gave blood at 13 and 19 months.

Nasopharyngeal and oropharyngeal swabs were obtained at each visit using transport swabs (nasopharyngeal - MW173 Amies transport medium, Transwab, Medical Wire and Equipment, Potley, UK; oropharyngeal - Culture-Swab Transport System, Venturi Transystem $^{\circledR}$, Copan Innovation, Italy). The swabs were kept at room temperature and processed within $16 \mathrm{~h}$. Results of the oropharyngeal swabs for positive pneumococcal culture were used only if the nasopharyngeal swabs were culture-negative. Pneumococcal identification and serotyping were performed as previously described [25].

\subsection{Serologic assays}

Serum serotype-specific pneumococcal anti-capsular IgG concentrations for PCV7 serotypes (4, 6A, 6B, 9V, 14, 18C, 19F and 23F) were measured using ELISA at the Department of Applied Immunology \& Endocrinology, Kinder und Jugendklinik Universitatsklinikum Erlangen, Germany, after double absorption with C-polysaccharide and pneumococcal serotype $22 \mathrm{~F}$ polysaccharide [5,26-28].

The opsonic activities of anti-pneumococcal antibodies were measured against PCV7 serotypes by a fourfold multiplexed opsonophagocytosis assay (MOPA) as previously described [29], with minor modifications [30].

Serum samples from which enough serum volume was available $(n=281)$ were submitted also to MOPA assay (in addition to ELISA assay). For those samples, ELISA GMCs were compared to MOPA GMTs. Since serotype 6A antigen is not a part of PCV7, serotype 6A MOPA was compared to serotype 6B ELISA antibodies.

\subsection{Data and statistical analysis}

Data were analyzed with SPSS 18.0 software for Windows (Chicago, IL, USA). Contingency table analyses were performed using $\chi^{2}$ test or Fisher's exact test. Continuous variables were analyzed using the $t$-test or ANOVA procedures. Correction for ethnicity 


\begin{tabular}{|c|c|c|c|c|c|c|c|c|c|c|c|c|c|c|c|c|c|c|c|c|c|c|c|c|}
\hline Age $(m)$ & 0 & 1 & 2 & 3 & 4 & 5 & 6 & 7 & 8 & \begin{tabular}{l|l}
9 & 1
\end{tabular} & $10 \mid 11$ & \begin{tabular}{l|l}
1 & 12
\end{tabular} & 13 & 14 & 15 & \begin{tabular}{l|l}
16 & 1
\end{tabular} & \begin{tabular}{l|l}
17 & 18 \\
$r$
\end{tabular} & \begin{tabular}{l|l}
3 & 19
\end{tabular} & 20 & 21 & 22 & 23 & 24 & 30 \\
\hline PCV7 3+1 & & & & & & & & & & & & & & & & & & & & & & & & \\
\hline PCV7 3+0 & & & & & & & & & & & & & & & & & & & & & & & & \\
\hline PCV7 0+2 & & & & & & & & & & & & & & & & & & & & & & & & \\
\hline Blood sample & & & $\sqrt{ }$ & & & & & $\sqrt{ }$ & & & & & $\sqrt{ }$ & & & & & $\sqrt{ }$ & & & & & & \\
\hline NP/OP culture & & & $\sqrt{ }$ & & $\sqrt{ }$ & & $\sqrt{ }$ & $\sqrt{ }$ & & & & $\sqrt{ }$ & $\sqrt{ }$ & & & & $\sqrt{ }$ & $\sqrt{ }$ & & & & & $\sqrt{ }$ & $\sqrt{ }$ \\
\hline Concomitant Vaccines & нви & HBv & 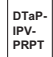 & & 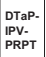 & & 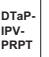 & нву & & & & 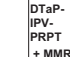 & & & & & HAV & & & & & & tav & \\
\hline
\end{tabular}

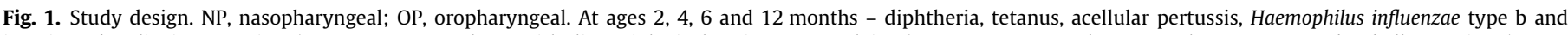

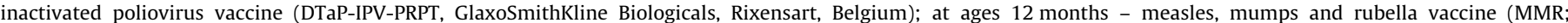

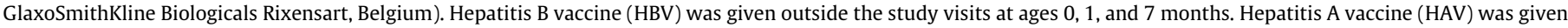
outside the study visits at ages 18 , and 24 months.

was done using Mantel-Haenszel or multi-regression analysis. A Pvalue $<.05$ was considered significant.

For the post-primary series, the proportion of subjects with serotype-specific IgG concentrations $\geq 0.35$ and $>1.0 \mu \mathrm{g} / \mathrm{mL}$ were calculated. For ages 13 and 19 months the proportion of subjects with concentration $>1.0$ and $>5.0 \mu \mathrm{g} / \mathrm{mL}$ were calculated. The latter was associated in previous publications with protection against carriage [31,32]. Additional comparisons between groups were conducted using $\log _{10}$-transformed geometric mean concentrations (GMCs).
Since protection against serotype $6 \mathrm{~A}$ may be obtained through antibodies elicited by serotype $6 \mathrm{~B}$ antigen [33], we combined serotype $6 \mathrm{~A}$ in the analysis with 7VT.

In a previous study [5], we demonstrated that although Bedouin infants had higher maternally-derived serotype-specific serum IgG than the Jewish infants for all serotypes, after the infant immunization series, no significant difference between the 2 ethnic groups was observed. Nevertheless, all serology results were adjusted for ethnic group. Furthermore, in another study, ethnicity did not

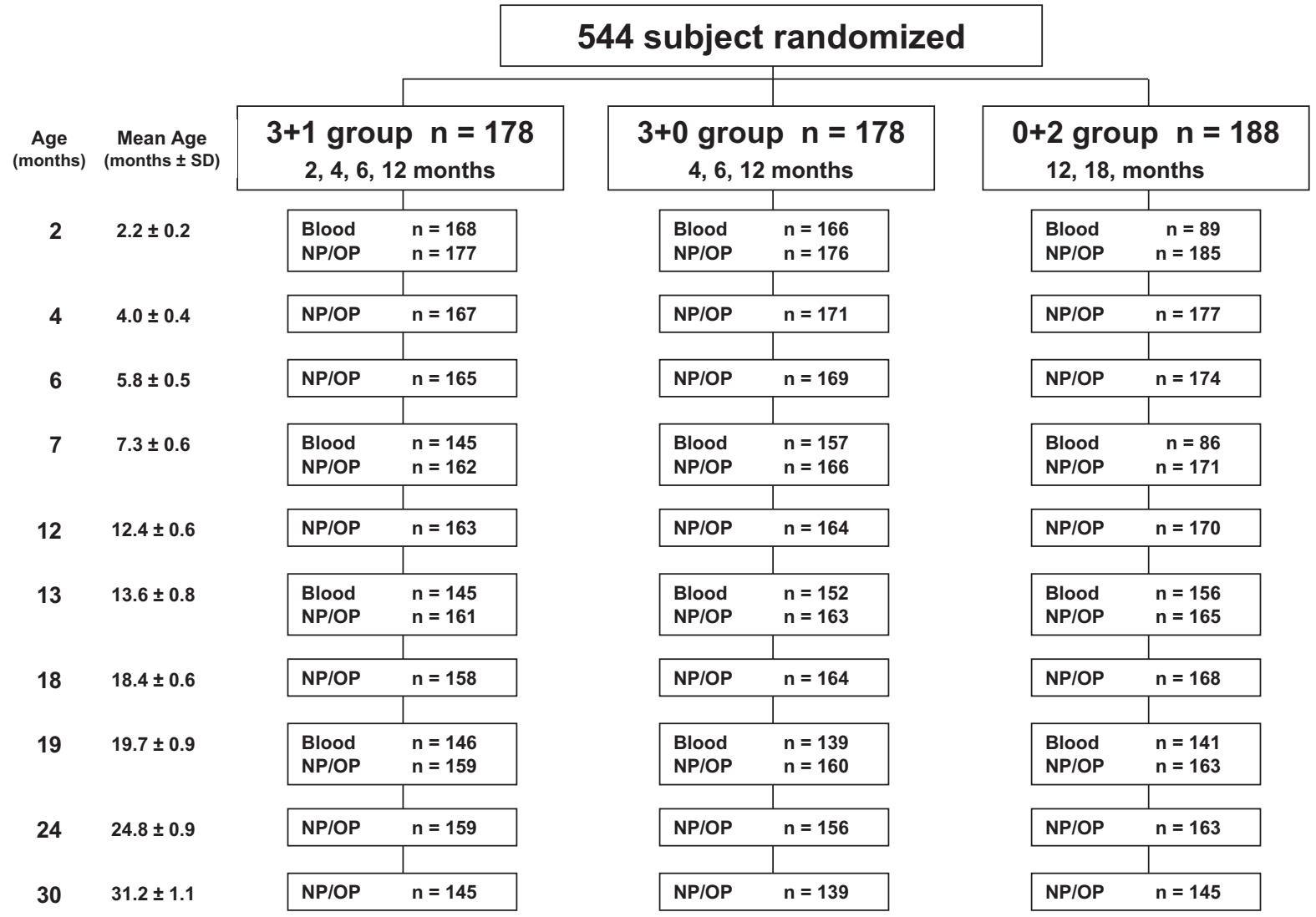

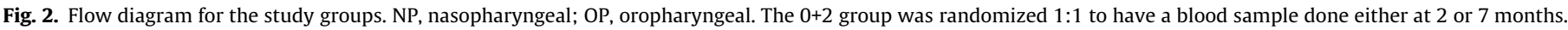
$\mathrm{SD}=$ Standard Deviation. 
Table 1

Serum serotype-specific pneumococcal anticapsular IgG GMCs $(\mu \mathrm{g} / \mathrm{mL})$ and percent of subjects achieving serum concentrations $>0.35,>1.0$ and $>5.0 \mu \mathrm{g} / \mathrm{mL}$ at 7,13 and $19 \mathrm{months}$ of age.

\begin{tabular}{|c|c|c|c|c|c|c|c|c|c|c|c|c|c|}
\hline \multirow[t]{2}{*}{ Serotype } & & \multicolumn{4}{|l|}{7 months } & \multicolumn{4}{|l|}{13 months } & \multicolumn{4}{|c|}{19 months } \\
\hline & & $\begin{array}{l}3+1 \\
\mathrm{n}=145\end{array}$ & $\begin{array}{l}3+0 \\
n=157\end{array}$ & $\begin{array}{l}0+2 \\
\mathrm{n}=86\end{array}$ & $P$ & $\begin{array}{l}3+1 \\
\mathrm{n}=145\end{array}$ & $\begin{array}{l}3+0 \\
n=152\end{array}$ & $\begin{array}{l}0+2 \\
\mathrm{n}=156\end{array}$ & $P$ & $\begin{array}{l}3+1 \\
\mathrm{n}=146\end{array}$ & $\begin{array}{l}3+0 \\
n=139\end{array}$ & $\begin{array}{l}0+2 \\
n=141\end{array}$ & $P^{*}$ \\
\hline \multirow[t]{4}{*}{4} & GMC (95\% CI) & $\begin{array}{l}1.9 \\
(1.6 ; 2.3)\end{array}$ & $\begin{array}{l}2.0 \\
(1.7 ; 2.2)\end{array}$ & $\begin{array}{l}0.0 \\
(0.0 ; 0.0)\end{array}$ & $\beta 2, \gamma 2$ & $\begin{array}{l}4.0 \\
(3.4 ; 4.7)\end{array}$ & $\begin{array}{l}0.3 \\
(0.3 ; 0.4)\end{array}$ & $\begin{array}{l}2.5 \\
(2.1 ; 3.1)\end{array}$ & $\alpha 2, \beta 2, \gamma 2$ & $\begin{array}{l}0.4 \\
(0.4 ; 0.5)\end{array}$ & $\begin{array}{l}0.1 \\
(0.1 ; 0.2)\end{array}$ & $\begin{array}{l}4.4 \\
(3.7 ; 5.3)\end{array}$ & $\alpha 2, \beta 2, \gamma 2$ \\
\hline & $\% \geq 0.35 \mu \mathrm{g} / \mathrm{mL}$ & 98.6 & 98.1 & 2.3 & $\beta 2, \gamma 2$ & ND & ND & ND & ND & $\mathrm{ND}$ & $\mathrm{ND}$ & ND & ND \\
\hline & $\%>1.0 \mu \mathrm{g} / \mathrm{mL}$ & 77.9 & 79.6 & 1.2 & $\beta 2, \gamma 2$ & 91.0 & 9.9 & 78.1 & $\alpha 2, \beta 1, \gamma 2$ & 81.4 & 46.8 & 100 & $\alpha 2, \beta 2, \gamma 1$ \\
\hline & $\%>5.0 \mu \mathrm{g} / \mathrm{mL}$ & ND & ND & $\mathrm{ND}$ & ND & 42.8 & 0.0 & 30.1 & $\alpha 2, \beta 1, \gamma 2$ & 19.9 & 10.8 & 89.4 & $\alpha 1, \beta 2, \gamma 2$ \\
\hline \multirow[t]{4}{*}{$6 B$} & GMC (95\% CI) & $\begin{array}{l}2.3 \\
(1.8 ; 3.0)\end{array}$ & $\begin{array}{l}1.9 \\
(1.4 ; 2.4)\end{array}$ & $\begin{array}{l}0.1 \\
(0.1 ; 0.1)\end{array}$ & $\beta 2, \gamma 2$ & $\begin{array}{l}11.0 \\
(8.8 ; 13.8)\end{array}$ & $\begin{array}{l}0.8 \\
(0.6 ; 1.0)\end{array}$ & $\begin{array}{l}0.4 \\
(0.3 ; 0.5)\end{array}$ & $\alpha 2, \beta 2, \gamma 2$ & $\begin{array}{l}2.0 \\
(1.6 ; 2.4)\end{array}$ & $\begin{array}{l}0.8 \\
(0.6 ; 0.9)\end{array}$ & $\begin{array}{l}2.9 \\
(2.3 ; 3.7)\end{array}$ & $\alpha 2, \beta 1, \gamma 2$ \\
\hline & $\% \geq 0.35 \mu \mathrm{g} / \mathrm{mL}$ & 89.7 & 83.4 & 8.1 & $\beta 2, \gamma 2$ & ND & $\mathrm{ND}$ & ND & ND & $\mathrm{ND}$ & $\mathrm{ND}$ & $\mathrm{ND}$ & ND \\
\hline & $\%>1.0 \mu \mathrm{g} / \mathrm{mL}$ & 72.9 & 70.7 & 1.2 & $\beta 2, \gamma 2$ & 93.1 & 40.8 & 25.2 & $\alpha 2, \beta 2, \gamma 1$ & 23.3 & 5.0 & 87.2 & $\alpha 2, \beta 2, \gamma 2$ \\
\hline & $\%>5.0 \mu \mathrm{g} / \mathrm{mL}$ & ND & ND & ND & ND & 79.3 & 9.2 & 2.6 & $\alpha 2, \beta 2, \gamma 1$ & 0.7 & 0.7 & 15.7 & $\beta 2, \gamma 2$ \\
\hline \multirow[t]{4}{*}{$9 \mathrm{~V}$} & GMC (95\% CI) & $\begin{array}{l}1.5 \\
(1.2 ; 1.7)\end{array}$ & $\begin{array}{l}1.4 \\
(1.3 ; 1.6)\end{array}$ & $\begin{array}{l}0.1 \\
(0.1 ; 0.1)\end{array}$ & $\beta 2, \gamma 2$ & $\begin{array}{l}3.5 \\
(3.0 ; 4.0)\end{array}$ & $\begin{array}{l}0.5 \\
(0.4 ; 0.5)\end{array}$ & $\begin{array}{l}2.0 \\
(1.7 ; 2.4)\end{array}$ & $\alpha 2, \beta 2, \gamma 2$ & $\begin{array}{l}0.7 \\
(0.6 ; 0.8)\end{array}$ & $\begin{array}{l}0.3 \\
(0.3 ; 0.4)\end{array}$ & $\begin{array}{l}3.6 \\
(3.0 ; 4.2)\end{array}$ & $\alpha 2, \beta 2, \gamma 2$ \\
\hline & $\% \geq 0.35 \mu \mathrm{g} / \mathrm{mL}$ & 96.6 & 96.2 & 4.7 & $\beta 2, \gamma 2$ & ND & ND & ND & ND & $\mathrm{ND}$ & $\mathrm{ND}$ & $\mathrm{ND}$ & ND \\
\hline & $\%>1.0 \mu \mathrm{g} / \mathrm{mL}$ & 64.1 & 66.0 & 2.3 & $\beta 2, \gamma 2$ & 92.4 & 16.4 & 78.7 & $\alpha 2, \beta 1, \gamma 2$ & 43.8 & 30.2 & 85.8 & $\alpha 1, \beta 2, \gamma 2$ \\
\hline & $\%>5.0 \mu \mathrm{g} / \mathrm{mL}$ & ND & ND & ND & ND & 33.1 & 0.0 & 18.1 & $\alpha 2, \beta 1, \gamma 2$ & 14.4 & 10.8 & 55.3 & $\beta 2, \gamma 2$ \\
\hline \multirow[t]{4}{*}{14} & GMC (95\% CI) & $\begin{array}{l}5.2 \\
(4.2 ; 6.5)\end{array}$ & $\begin{array}{l}5.0 \\
(4.2 ; 6.1)\end{array}$ & $\begin{array}{l}0.1 \\
(0.0 ; 0.1)\end{array}$ & $\beta 2, \gamma 2$ & $\begin{array}{l}12.9 \\
(11.0 ; 15.2)\end{array}$ & $\begin{array}{l}1.4 \\
(1.1 ; 1.7)\end{array}$ & $\begin{array}{l}2.1 \\
(1.7 ; 2.6)\end{array}$ & $\alpha 2, \beta 2, \gamma 1$ & $\begin{array}{l}2.4 \\
(2.0 ; 2.8)\end{array}$ & $\begin{array}{l}0.9 \\
(0.7 ; 1.1)\end{array}$ & $\begin{array}{l}15.4 \\
(13.2 ; 17.9)\end{array}$ & $\alpha 2, \beta 2, \gamma 2$ \\
\hline & $\% \geq 0.35 \mu \mathrm{g} / \mathrm{mL}$ & 93.8 & 95.5 & 20.9 & $\beta 2, \gamma 2$ & ND & ND & ND & ND & ND & ND & ND & ND \\
\hline & $\%>1.0 \mu \mathrm{g} / \mathrm{mL}$ & 88.3 & 87.3 & 4.7 & $\beta 2, \gamma^{2}$ & 98.6 & 61.2 & 73.1 & $\alpha 2, \beta 2, \gamma 1$ & 46.9 & 8.6 & 80.7 & $\alpha 2, \beta 2, \gamma 2$ \\
\hline & $\%>5.0 \mu \mathrm{g} / \mathrm{mL}$ & ND & ND & ND & ND & 84.1 & 11.2 & 23.7 & $\alpha 2, \beta 2, \gamma 1$ & 6.2 & 0.7 & 27.7 & $\alpha 1, \beta 2, \gamma 2$ \\
\hline \multirow[t]{4}{*}{$18 \mathrm{C}$} & GMC (95\% CI) & $\begin{array}{l}1.5 \\
(1.3 ; 1.8)\end{array}$ & $\begin{array}{l}1.7 \\
(1.5 ; 1.9)\end{array}$ & $\begin{array}{l}0.0 \\
(0.0 ; 0.0)\end{array}$ & $\beta 2, \gamma 2$ & $\begin{array}{l}3.7 \\
(3.2 ; 4.3)\end{array}$ & $\begin{array}{l}0.3 \\
(0.3 ; 0.4)\end{array}$ & $\begin{array}{l}1.9 \\
(1.6 ; 2.3)\end{array}$ & $\alpha 2, \beta 2, \gamma 2$ & $\begin{array}{l}0.5 \\
(0.4 ; 0.6)\end{array}$ & $\begin{array}{l}0.2 \\
(0.2 ; 0.2)\end{array}$ & $\begin{array}{l}2.3 \\
(2.0 ; 2.7)\end{array}$ & $\alpha 2, \beta 2, \gamma 2$ \\
\hline & $\% \geq 0.35 \mu \mathrm{g} / \mathrm{mL}$ & 93.8 & 98.7 & 1.2 & $\alpha 1, \beta 2, \gamma 2$ & ND & ND & ND & ND & ND & ND & ND & ND \\
\hline & $\%>1.0 \mu \mathrm{g} / \mathrm{mL}$ & 72.7 & 73.5 & 1.2 & $\beta 2, \gamma 2$ & 91.0 & 8.6 & 75.0 & $\alpha 2, \beta 2, \gamma 2$ & 15.8 & 1.4 & 91.4 & $\alpha 2, \beta 2, \gamma 2$ \\
\hline & $\%>5.0 \mu \mathrm{g} / \mathrm{mL}$ & ND & ND & ND & ND & 38.6 & 0.7 & 18.6 & $\alpha 2, \beta 2, \gamma 2$ & 2.7 & 0.7 & 52.5 & $\beta 2, \gamma 2$ \\
\hline \multirow[t]{4}{*}{$19 \mathrm{~F}$} & GMC (95\% CI) & $\begin{array}{l}1.8 \\
(1.5 ; 2.2)\end{array}$ & $\begin{array}{l}2.1 \\
(1.8 ; 2.5)\end{array}$ & $\begin{array}{l}0.0 \\
(0.0 ; 0.0)\end{array}$ & $\beta 2, \gamma 2$ & $\begin{array}{l}4.1 \\
(3.4 ; 4.9)\end{array}$ & $\begin{array}{l}0.5 \\
(0.4 ; 0.7)\end{array}$ & $\begin{array}{l}0.4 \\
(0.4 ; 0.5)\end{array}$ & $\alpha 2, \beta 2$ & $\begin{array}{l}1.0 \\
(0.8 ; 1.3)\end{array}$ & $\begin{array}{l}0.6 \\
(0.5 ; 0.8)\end{array}$ & $\begin{array}{l}5.0 \\
(3.9 ; 6.3)\end{array}$ & $\alpha 1, \beta 2, \gamma 2$ \\
\hline & $\% \geq 0.35 \mu \mathrm{g} / \mathrm{mL}$ & 91 & 94.9 & 1.2 & $\beta 2, \gamma 2$ & ND & ND & ND & ND & $\mathrm{ND}$ & ND & $\mathrm{ND}$ & ND \\
\hline & $\%>1.0 \mu \mathrm{g} / \mathrm{mL}$ & 74.5 & 78.3 & 1.2 & $\beta 2, \gamma 2$ & 89.0 & 23.0 & 26.9 & $\alpha 2, \beta 2$ & 73.1 & 37.0 & 75.2 & $\alpha 2, \gamma 2$ \\
\hline & $\%>5.0 \mu \mathrm{g} / \mathrm{mL}$ & ND & ND & ND & $\mathrm{ND}$ & 46.2 & 8.6 & 3.2 & $\alpha 2, \beta 2, \gamma 1$ & 19.9 & 7.2 & 39.7 & $\alpha 1, \beta 2, \gamma 2$ \\
\hline \multirow[t]{5}{*}{$23 \mathrm{~F}$} & GMC (95\% CI) & $\begin{array}{l}1.3 \\
(1.0 ; 1.5)\end{array}$ & $\begin{array}{l}1.0 \\
(0.9 ; 1.3)\end{array}$ & $\begin{array}{l}0.1 \\
(0.0 ; 0.1)\end{array}$ & $\beta 2, \gamma 2$ & $\begin{array}{l}5.6 \\
(4.7 ; 6.7)\end{array}$ & $\begin{array}{l}0.4 \\
(0.3 ; 0.5)\end{array}$ & $\begin{array}{l}0.6 \\
(0.5 ; 0.7)\end{array}$ & $\alpha 2, \beta 2, \gamma 1$ & $\begin{array}{l}0.9 \\
(0.7 ; 1.1)\end{array}$ & $\begin{array}{l}0.3 \\
(0.2 ; 0.3)\end{array}$ & 2.8 & $\alpha 2, \beta 2, \gamma 2$ \\
\hline & & & & & & & & & & & & $(2.2 ; 3.4)$ & \\
\hline & $\% \geq 0.35 \mu \mathrm{g} / \mathrm{mL}$ & 88.3 & 82.2 & 4.7 & $\beta 2, \gamma 2$ & ND & ND & ND & ND & ND & ND & ND & ND \\
\hline & $\%>1.0 \mu \mathrm{g} / \mathrm{mL}$ & 57.6 & 51.9 & 2.3 & $\beta 2, \gamma 2$ & 95.9 & 19.7 & 32.3 & $\alpha 2, \beta 2, \gamma 1$ & 29.5 & 8.6 & 91.4 & $\alpha 2, \beta 2, \gamma 2$ \\
\hline & $\%>5.0 \mu \mathrm{g} / \mathrm{mL}$ & ND & ND & ND & ND & 54.9 & 1.3 & 5.1 & $\alpha 2, \beta 2$ & 2.7 & 1.4 & 34.0 & $\beta 2, \gamma 2$ \\
\hline
\end{tabular}

$\alpha, 3+1$ vs $3+0$ group: $\alpha_{1}, .001 \leq P<.05 ; \alpha_{2}, P<.001$.

$\beta, 3+1$ vs $0+2$ group: $\beta_{1}, .001 \leq P<.05 ; \beta_{2}, P<.001$

$\gamma, 3+0$ vs $0+2$ group: $\gamma_{1}, .001 \leq P<.05 ; \gamma_{2}, P<.001$

$\mathrm{ND}=$ Not done.

$P$ value adjusted for ethnic group. 
influence the association between $\operatorname{Ig} G$ concentrations and prevention of carriage despite differences in carriage rates [34].

New pneumococcal acquisition was defined as acquisition of a serotype not seen at any previous visit. New acquisitions were calculated from 7 months of age (one month following complete infant series) onwards. Cumulative incidences were calculated by adding the incidence of a certain point (adjusted for ethnicity) to all previous rates within the same group. All acquisitions and prevalence rates are expressed per 100 children.

\section{Results}

Overall, 3100 eligible infants were invited to take part in the study. The parents of 1141 were willing to consider participation in the study, and 544 agreed to be enrolled: $3+1(n=178), 3+0$ $(\mathrm{n}=178)$ and $0+2(\mathrm{n}=188)$ (Fig. 2). At all visits, the mean age was similar in all groups. Bedouin infants constituted $50 \%$ and females constituted $48 \%$ of subjects, with no significant differences in these distributions between the $3+1,3+0$ and $0+2$ groups.

\subsection{Immunogenicity}

At 7 months of age, no significant differences in immunogenicity between the $3+0$ and $3+1$ groups were observed, except for serotype $18 \mathrm{C}$, with a higher proportion of infants with antibody concentrations $\geq 0.35 \mu \mathrm{g} / \mathrm{ml}$ in the $3+0$ group (Table 1 ). For all serotypes, the antibody concentrations were higher among both $3+1$ and $3+0$ groups compared to the $0+2$ group.
At 13 months, the antibody concentrations were significantly higher in the $3+1$ than in the $3+0$ group for all serotypes. The 0 +2 group (after 1 dose at 12 months) had higher antibody concentrations than the $3+0$ group for all serotypes, except $19 \mathrm{~F}$ and $6 \mathrm{~B}$ and lower antibody concentrations than the $3+1$ group for all serotypes. At age 19 months, one month after the second PCV7 dose for group $0+2$, the antibody concentrations in the $0+2$ group were higher for all serotypes compared to both $3+1$ and $3+0$ groups. The concentrations in the $3+1$ group were all significantly higher than those in the $3+0$ group.

Trends for ELISA GMCs paralleled those of MOPA GMTs for each serotype and study age group (Fig. 3).

\subsection{Nasopharyngeal/oropharyngeal new acquisition rates}

Overall, nasopharyngeal/oropharyngeal (NP/OP) cultures samples were obtained in 4925 visits: 1616, 1628 and 1681 for the 3 $+1,3+0$, and $0+2$ groups, respectively (Fig. 2). Cultures were positive for S. pneumoniae in 2673 (54.3\%) visits; of these, $0.6 \%$ were positive from the OP sample only. A total of 496 (10.1\%) new acquisitions of 7VT+6A were observed: $6 \mathrm{~A}(123,2.5 \%), 19 \mathrm{~F}(112,2.4 \%)$ ), $6 \mathrm{~B}(78,1.6 \%), 23 \mathrm{~F}(77,1.6 \%), 14$ (55, 1.1\%), 18C (25, 0.5\%), 9V (20, $0.4 \%)$ and $4(6,0.1 \%)$. Serotypes $6 \mathrm{~A}$ and $6 \mathrm{~B}$ constituted together $40.5 \%$ of all new acquisitions.

\subsubsection{Comparison between the $3+1$, the $3+0$ and the $0+2$ groups}

Acquisition of $7 \mathrm{VT}+6 \mathrm{~A}$ (combined) was similar for groups $3+1$ and $3+0$ at all ages from 7 to 30 months, with no significant differences in any of the age points (Fig. 4A. P-values in Fig. 4 reflect the
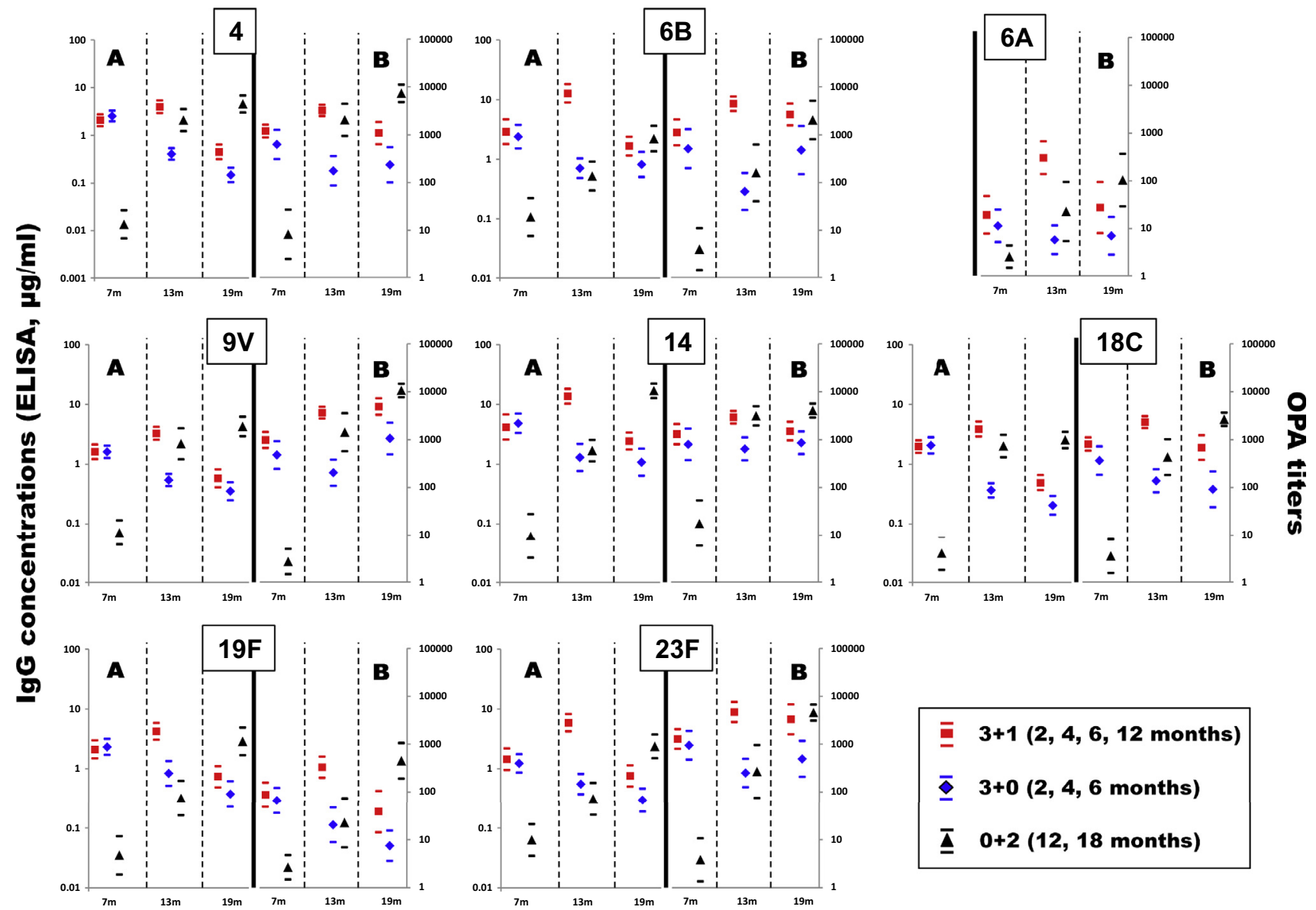

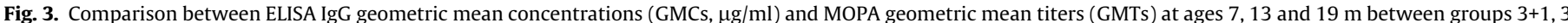

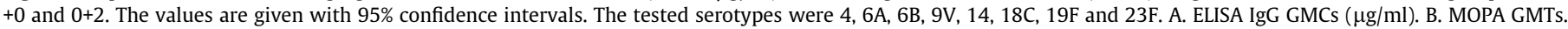
"Since serotype 6A antigen is not a part of PCV7, serotype 6A MOPA GMTs were compared to serotype 6B ELISA GMCs. Statistical analyses are presented in Table 1. 
$0+2(12,18 \mathrm{~m})$

A

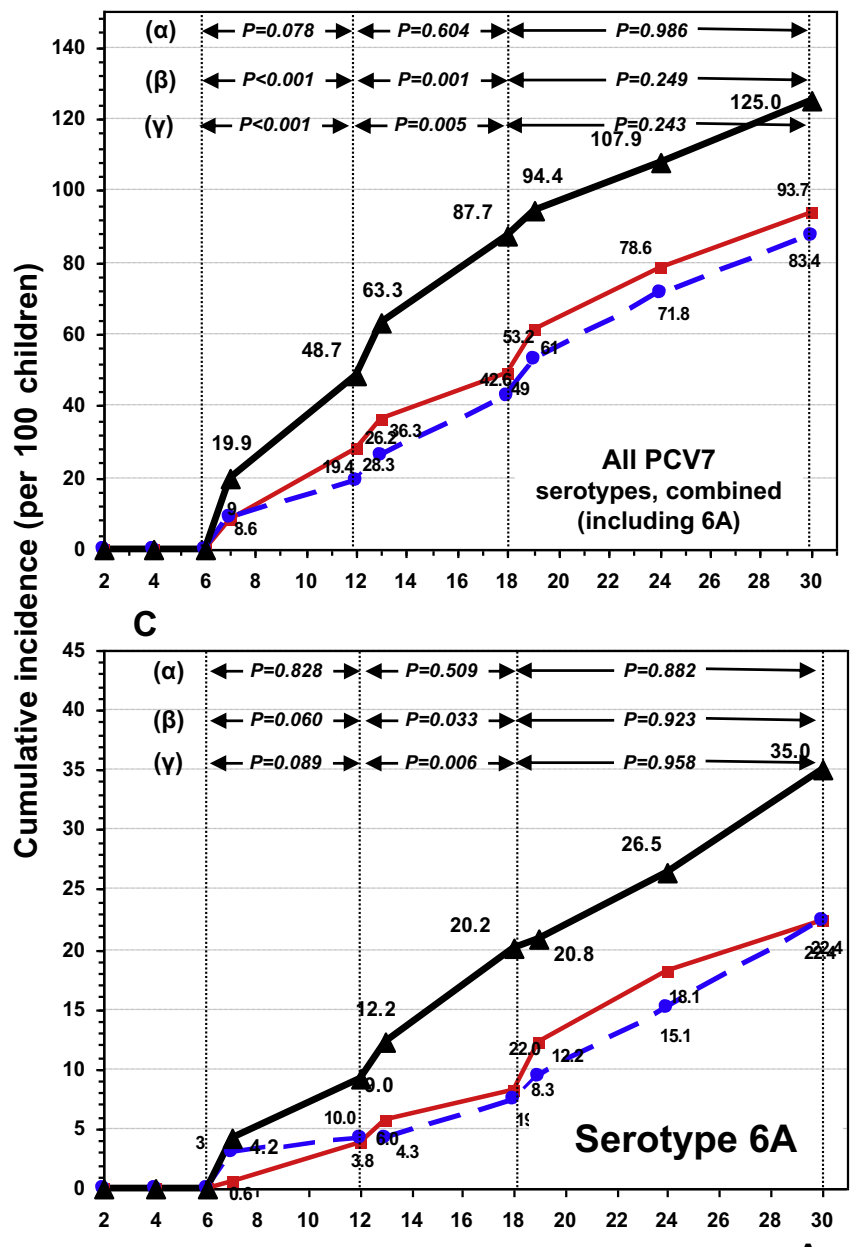

$3+1(2,4,6,12 m)--\rightarrow--3+0(2,4,6 m)$

B

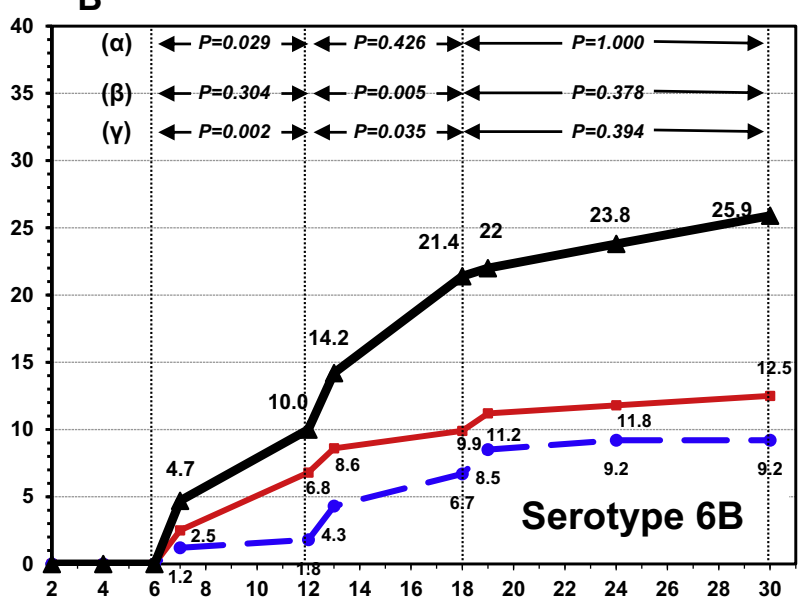

D

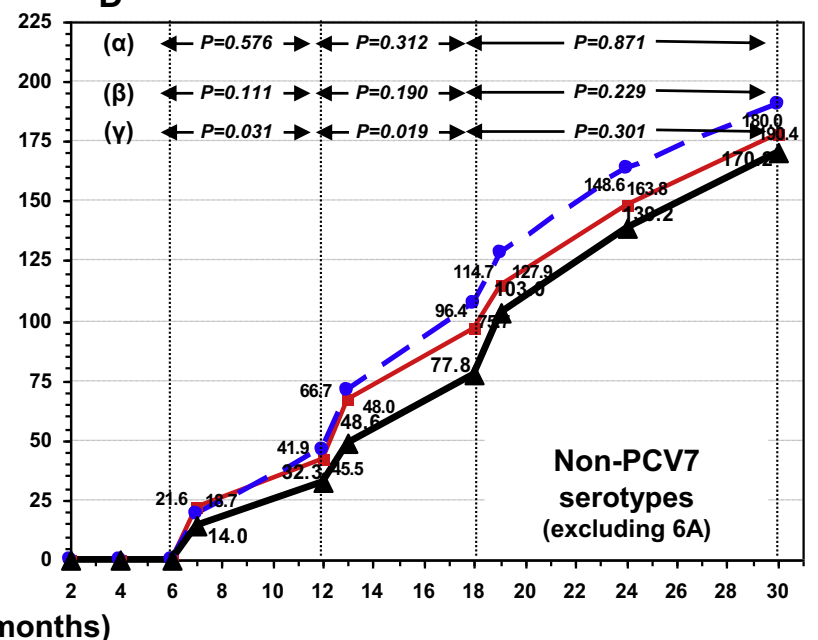

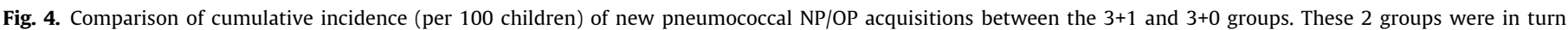

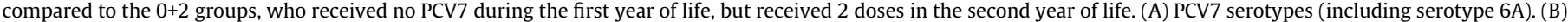

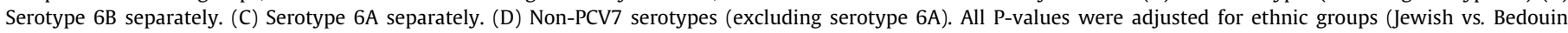

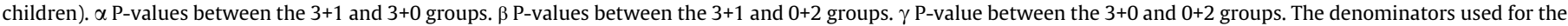
calculation of the incidence rates presented in Fig. 4 are detailed in Fig. 2.

rate ratio (adjusted for ethnicity) between age-specific cumulative incidences.). Both groups differed significantly from the $0+2$ group at ages 7-12 months (a period during which no dose was administered to the $0+2$ group), and ages 13-18 months (after 1 dose at 12 months among the 0+2 group). However, at ages 19-30 months (after group $0+2$ received 2 PCV doses), no significant differences between the $0+2$ groups and the $3+1$ or $3+0$ group were observed. Several differences were observed for serotypes $6 B$ separately and $6 \mathrm{~A}$ separately (Fig. $4 \mathrm{~B}$ and $\mathrm{C}$ ). These include higher cumulative incidences of serotype $6 \mathrm{~B}$ in the $0+2$ group compared with the $3+0$ group at ages $7-12$ months and the $3+1$ and $3+0$ groups at ages 13-18 months. Similarly, for serotype 6A, higher cumulative incidences in the $0+2$ group were observed compared with the $3+1$ and $3+0$ groups at ages $13-18$ months. For non $7 \mathrm{VT}+6 \mathrm{~A}$ serotypes, no difference was observed between groups $3+1$ and $3+0$, but for the $3+0$ group acquisitions of non VT7 at ages 7-18 months was significantly higher than the group $0+2$ (Fig. 4D).

\subsection{Prevalence rates of nasopharyngeal/oropharyngeal carriage}

At any time point, and for all comparisons, no significant differences between groups $3+1$ and $3+0$ in the mean carriage prevalence were observed (Fig. 5). However, for all V7T+6A combined, and serotypes $6 \mathrm{~B}$ and $6 \mathrm{~A}$ separately, the mean carriage prevalence in groups $3+0$ and $3+1$ was generally lower than that in the $0+2$ group. This difference was statistically significant at ages $7+12$ and $13+8$ months for $7 \mathrm{VT}+6 \mathrm{~A}$ combined; all ages for serotype $6 \mathrm{~B}$; and age $13+18$ months for serotype $6 \mathrm{~A}$.

For non-VT+6A, the mean carriage prevalence was significantly higher in the $3+1$ and $3+0$ groups than in the $0+2$ group at ages 7 +12 and 13+18 months. No significant differences were observed for overall pneumococcal carriage prevalence between all groups.

\section{Discussion}

In the current study, when PCV7 was administered to infants at a 3-dose schedule with a 2-month interval (2, 4 and 6 months of age), adding a further dose at 12 months of age markedly and significantly boosted serotype-specific anticapsular IgG responses to all 7 serotypes and OPA titers to all measured serotypes, resulting in higher concentrations than without a booster for at least 6 months. However, paradoxically, no significant added benefit was observed in term of both nasopharyngeal acquisition and preva- 

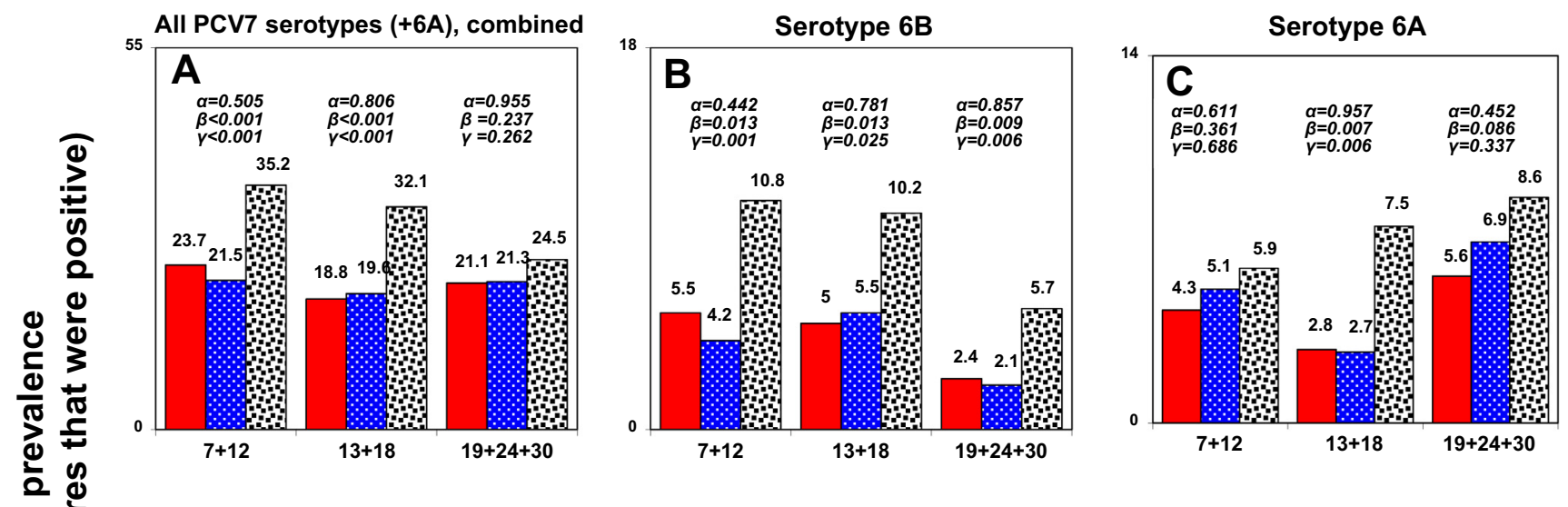

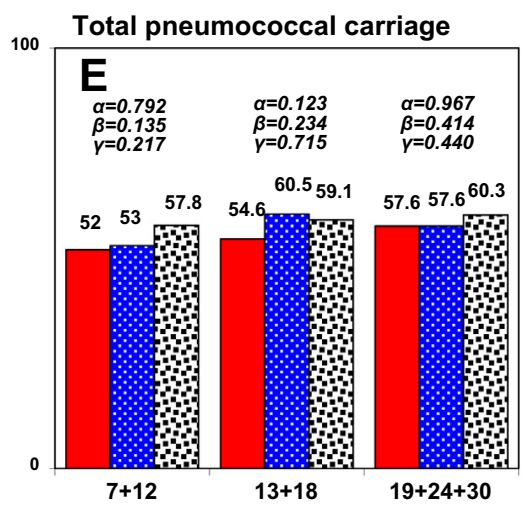

Age (months)

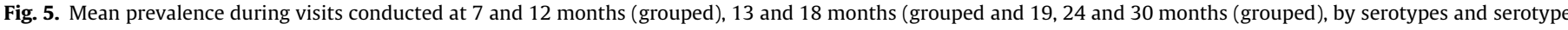

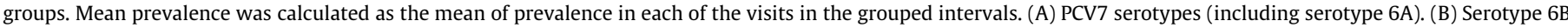

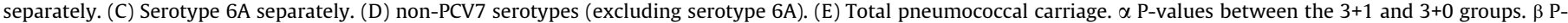
values between the $3+1$ and $0+2$ groups. $\gamma$ P-value between the $3+0$ and $0+2$ groups.

lence of VT7+6A serotypes throughout all visits up to 30 months of age. To the best of our knowledge, this is the only study that compared the efficacy of $3+0$ vs. $3+1$ regimens on carriage with a relatively long follow-up (age 2.5 years).

These findings are unique for several reasons. First, while $3+0$ PCV schedules were studied in several countries worldwide and both efficacy/effectiveness against both invasive pneumococcal disease [22] and nasopharyngeal carriage [35], and impact on these outcomes were shown, none of these studies were compared headto-head to the $3+1$ regimen. To our knowledge, this is the first study that compared $3+1$ and $3+0$ schedules within a comparative randomized trial. Furthermore, the study was conducted in Israel during a period when PCVs were not part of the NIP. This is of crucial importance, since without such a head-to-head study, it is difficult to assess the real difference in efficacy of the different schedules against nasopharyngeal carriage. Second, our findings are reassuring and suggest that reduced schedules may impact equally both direct and indirect protection through reduced VT7 spread. Reduced schedules are indeed desirable for several reasons, including the high cost of PCVs and to limit the growing number of injections in infant vaccination. Third, while a higher nasopharyngeal carriage rate with decreasing number of PCV doses in infants was observed in several studies $[8,12,13]$, our results demonstrate that the number of doses may play a smaller role than previously thought in reducing carriage, at least in a schedule with a 2- month interval between doses. Whether similar results can be obtained with a shorter interval between doses (i.e. the $6,10,14$ week interval used in developed countries), is not clear.

The lack of direct correlation between the post-booster serum antibody and carriage acquisition and prevalence is somewhat surprising. The following can be a plausible explanation. Correlation between serotype-specific $\operatorname{IgG}$ concentrations and nasopharyngeal acquisition of pneumococcus post infant 3-dose primary series was clearly demonstrated $[33,34]$. It may be speculated that high blood IgG concentration is not, by itself, sufficient for the prevention of carriage acquisition. Protection against carriage may require an additional stimulation of the immune system, building a higher number of memory B-cells. This hypothesis lends support from a recent human challenge study demonstrating that indeed, serotype-specific blood memory B-cells correlated with nasopharyngeal carriage, while circulating serotype-specific IgG or IgA levels or even functional capacity of serum antibody were not associated with protection against carriage [36]. In this case, we can speculate that PCV7 sufficiently stimulated specific memory B cells after 3 doses to protect against nasopharyngeal carriage acquisition of vaccine-serotype pneumococcus. Adding booster at 12 months of age, although resulting in boosting circulating antibody concentration, was not needed in the presence of the critical number of memory B cells. In our study, the protection against carriage without the booster was similar to that of the booster up to age 30 
months, and we did not examine further durations of protection. It is thus not clear whether the booster dose further stimulated the memory B-cell potentially resulting in longer protection than without a booster. This necessitates a longer follow-up, with additional studies needed measuring both B cell memory and antibodies concentration in correlation to protection against carriage for longer durations.

Other than the differences in the immunogenicity of the $3+1$ and $3+0$ schedules, one additional major concern regarding the use of reduced schedules is the possibility of lower indirect (herd) protection, as was observed in Australia compared to the United Kingdom and the United States, following PCV13 implementation [22]. The study raised questions about the serotypes included PCV13 beyond those in PCV7 and whether the longevity of protection against carriage of the additional PCV13 serotypes could not be shorter than that of the PCV7 serotypes. Since the key for successful herd protection is the reduction of new carriage acquisition, the question raised by the Australian experience emphasized that choosing $3+0$ over $3+1$ schedule should be accompanied with strict monitoring of pneumococcal disease rates in both vaccinated and non-vaccinated populations (both children $>5$ years and adults). Indeed, a transient increase in non-PCV7 serotypes carriage rate was observed in our study in the $3+0$ vs. the $0+2$ schedules (but not when the $3+1$ and the $0+2$ schedules were compared), again emphasizing the importance of strict monitoring when choosing $3+0$ over $3+1$ schedule.

The data from our controls (the $0+2$ schedule) are important. In the $0+2$ group, antibodies concentration and carriage acquisition rates were favorable with the $3+1$ and the $3+0$ groups, when evaluated in the 2 nd and the 3rd years of life. However, in the $0+2$ schedule, infants ( $<12$ months) are not directly protected against vaccine-type acquisition of carriage. This point is important in view of recent thought to potentially implement regimens with only 1 dose in infancy and a second later in life (booster at 9 months of age), relying on herd protection derived from reduction of cases.

The main strengths of our study include the relatively large number of subjects, the methodology of randomized, controlled trial and the fact that the study was conducted in the pre-PCV licensure era. Additionally, the relatively long-term follow-up for carriage enabled appreciation of the efficacy up to 2.5 years post the last dose of the $3+0$ regimen.

The main limitation of the current study is that only PCV7+6A serotypes were evaluated (rather than PCV13 or PCV10 serotypes, currently in use).

In conclusion, while a toddler booster PCV7 dose increased antibody concentrations, in the currently tested regimen of 2,4 and 6 months, the added benefit of the booster dose to reduce carriage of PCV7+6A serotypes was not shown. This suggests that protection against nasopharyngeal acquisition of $S$. pneumoniae does not necessarily depend on circulating IgG, but may also depend on other factors, such as the presence of circulating or submucosal memory B-cells.

\section{Source of support}

The study was funded in part by a grant from Pfizer [grant no. 0887X1-4603].

\section{Financial disclosure}

Ron Dagan has received grants/research support from Pfizer and Merck Sharp \& Dohme; has been a scientific consultant for MeMed, Merck Sharp \& Dohme, and Pfizer and a speaker for Pfizer.

Shalom Ben-Shimol has received speakers' fees from Pfizer.
Birgit Simell has no conflicts of interest to declare.

David Greenberg has received grants from Merck Sharp \& Dohme; has been a scientific consultant and a speaker for Merck Sharp \& Dohme and Pfizer.

Nurith Porat has no conflicts of interest to declare.

Helena Käyhty has no conflicts of interest to declare.

Noga Givon-Lavi has no conflicts of interest to declare.

\section{References}

[1] Whitney CG, Farley MM, Hadler J, Harrison LH, Bennett NM, Lynfield R, et al. Decline in invasive pneumococcal disease after the introduction of proteinpolysaccharide conjugate vaccine. N Engl J Med 2003;348(18):1737-46.

[2] De Carvalho Gomes H, Muscat M, Monnet DL, Giesecke J, Lopalco PL. Use of seven-valent pneumococcal conjugate vaccine (PCV7) in Europe, 2001-2007. Euro Surveill 2009;14(12).

[3] Whitney CG, Goldblatt D, O'Brien KL. Dosing schedules for pneumococcal conjugate vaccine: considerations for policy makers. Pediatr Infect Dis J 2014;33(Suppl 2):S172-81.

[4] Pneumococcal vaccines WHO position paper. 2012. Week Epidemiol Rec 2012;87:129-44

[5] Givon-Lavi N, Greenberg D, Dagan R. Immunogenicity of alternative regimens of the conjugated 7-valent pneumococcal vaccine - a randomized controlled trial. Pediatr Infect Dis J 2010;29(8):756-62.

[6] Fritzell B, Fletcher MA. Pneumococcal polysaccharide-protein (CRM197) conjugate vaccines, 7- or 9-valent, in the $2+1$ schedule. Expert Rev Vaccines 2011;10(3):263-90.

[7] van Gils EJ, Veenhoven RH, Hak E, Rodenburg GD, Bogaert D, Ijzerman EP, et al. Effect of reduced-dose schedules with 7-valent pneumococcal conjugate vaccine on nasopharyngeal pneumococcal carriage in children: a randomized controlled trial. JAMA 2009;302(2):159-67.

[8] Russell FM, Carapetis JR, Satzke C, Tikoduadua L, Waqatakirewa L, Chandra R, et al. Pneumococcal nasopharyngeal carriage following reduced doses of a 7 valent pneumococcal conjugate vaccine and a 23-valent pneumococcal polysaccharide vaccine booster. Clin Vaccine Immunol 2010;17(12):1970-6.

[9] Russell FM, Balloch A, Tang ML, Carapetis JR, Licciardi P, Nelson J, et al. Immunogenicity following one, two, or three doses of the 7-valent pneumococcal conjugate vaccine. Vaccine 2009;27(41):5685-91.

[10] Scott P, Rutjes AW, Bermetz L, Robert N, Scott S, Lourenco T, et al. Comparing pneumococcal conjugate vaccine schedules based on 3 and 2 primary doses: systematic review and meta-analysis. Vaccine 2011;29(52):9711-21.

[11] Ruckinger S, Dagan R, Albers L, Schonberger K, von Kries R. Immunogenicity of pneumococcal conjugate vaccines in infants after two or three primary vaccinations: a systematic review and meta-analysis. Vaccine 2011;29 (52):9600-6.

[12] Dagan R, Givon-Lavi N, Porat N, Greenberg D. The effect of an alternative reduced-dose infant schedule and a second year catch-up schedule with 7valent pneumococcal conjugate vaccine on pneumococcal carriage: a randomized controlled trial. Vaccine 2012;30(34):5132-40.

[13] Ota MO, Akinsola A, Townend J, Antonio M, Enwere G, Nsekpong D, et al. The immunogenicity and impact on nasopharyngeal carriage of fewer doses of conjugate pneumococcal vaccine immunization schedule. Vaccine 2011;29 (16):2999-3007.

[14] Conklin L, Deloria Knoll M, Loo JD, Fleming-Dutra KE, Park DE, Scott Johnson T, et al. Landscape analysis of pneumococcal conjugate vaccine dosing schedules: a systematic review. Sub-report on the 3-dose schedules. A project of the AVI technical assistance consortium (AVI-TAC) Final report 1.0, 17 October 2011. <http://www.who.int/immunization/sage/3_Conklin_L_PCV_Dosing Landscape_Report_Oct_17_2011_FINAL_nov11.pdf> [accessed December 2017].

[15] Azzari C, Resti M. Reduction of carriage and transmission of Streptococcus pneumoniae: the beneficial "side effect" of pneumococcal conjugate vaccine. Clin Infect Dis 2008;47(8):997-9.

[16] Kayhty H, Auranen K, Nohynek H, Dagan R, Makela H. (PneumoCarr) atPCG. Nasopharyngeal colonization: a target for pneumococcal vaccination. Expert Rev Vaccines 2006;5(5):651-68.

[17] Pilishvili T, Lexau C, Farley MM, Hadler J, Harrison LH, Bennett NM, et al. Sustained reductions in invasive pneumococcal disease in the era of conjugate vaccine. J Infect Dis 2010;201(1):32-41.

[18] Mbelle N, Huebner RE, Wasas AD, Kimura A, Chang I, Klugman KP. Immunogenicity and impact on nasopharyngeal carriage of a nonavalent pneumococcal conjugate vaccine. J Infect Dis 1999;180(4):1171-6.

[19] Roca A, Hill PC, Townend J, Egere U, Antonio M, Bojang A, et al. Effects of community-wide vaccination with PCV-7 on pneumococcal nasopharyngeal carriage in the Gambia: a cluster-randomized trial. PLoS Med 2011;8(10): e1001107.

[20] Madhi SA, Adrian P, Kuwanda L, Cutland C, Albrich WC, Klugman KP. Longterm effect of pneumococcal conjugate vaccine on nasopharyngeal colonization by Streptococcus pneumoniae-and associated interactions with Staphylococcus aureus and Haemophilus influenzae colonization-in HIVinfected and HIV-uninfected children. J Infect Dis 2007;196(11):1662-6. 
[21] Roche PW, Krause V, Cook H, Barralet J, Coleman D, Sweeny A, et al. Invasive pneumococcal disease in Australia, 2006. Commun Dis Intell 2008;32 (1):18-30

[22] Jayasinghe S, Menzies R, Chiu C, Toms C, Blyth CC, Krause V, et al. Long-term impact of a " $3+0$ " schedule for 7 - and 13-valent pneumococcal conjugate vaccines on invasive pneumococcal disease in Australia, 2002-2014. Clin Infect Dis 2017;64(2):175-83.

[23] Statistical Abstracts of Israel, No. 49. Central Bureau of Statistics. Jerusalem: Hemed Press Ltd.; 1998.

[24] Ben-Shimol S, Givon-Lavi N, Greenberg D, Dagan R. Pneumococcal nasopharyngeal carriage in children $<5$ years of age visiting the pediatric emergency room in relation to PCV7 and PCV13 introduction in southern Israel. Hum Vaccin Immunother 2016;12(2):268-76.

[25] Barkai G, Greenberg D, Givon-Lavi N, Dreifuss E, Vardy D, Dagan R. Community prescribing and resistant Streptococcus pneumoniae. Emerg Infect Dis 2005;11 (6):829-37.

[26] Quataert SA, Kirch CS, Wiedl LJ, Phipps DC, Strohmeyer S, Cimino CO, et al. Assignment of weight-based antibody units to a human antipneumococcal standard reference serum, lot 89-S. Clin Diagn Lab Immunol 1995;2(5):590-7.

[27] Concepcion NF, Frasch CE. Pneumococcal type $22 \mathrm{f}$ polysaccharide absorption improves the specificity of a pneumococcal-polysaccharide enzyme-linked immunosorbent assay. Clin Diagn Lab Immunol 2001;8(2):266-72.

[28] Quataert SA, Rittenhouse-Olson K, Kirch CS, Hu B, Secor S, Strong N, et al. Assignment of weight-based antibody units for 13 serotypes to a human antipneumococcal standard reference serum, lot 89-S(f). Clin Diagn Lab Immunol 2004;11(6):1064-9.

[29] Burton RL, Nahm MH. Development and validation of a fourfold multiplexed opsonization assay (MOPA4) for pneumococcal antibodies. Clin Vaccine Immunol 2006;13(9):1004-9.
[30] Simell B, Vuorela A, Ekstrom N, Palmu A, Reunanen A, Meri S, et al. Aging reduces the functionality of anti-pneumococcal antibodies and the killing of Streptococcus pneumoniae by neutrophil phagocytosis. Vaccine 2011;29 (10):1929-34.

[31] Goldblatt D, Hussain M, Andrews N, Ashton L, Virta C, Melegaro A, et al. Antibody responses to nasopharyngeal carriage of Streptococcus pneumoniae in adults: a longitudinal household study. J Infect Dis 2005;192(3):387-93.

[32] Millar EV, O’Brien KL, Bronsdon MA, Madore D, Hackell JG, Reid R, et al. Anticapsular serum antibody concentration and protection against pneumococcal colonization among children vaccinated with 7-valent pneumococcal conjugate vaccine. Clin Infect Dis 2007;44(9):1173-9.

[33] Dagan R, Givon-Lavi N, Fraser D, Lipsitch M, Siber GR, Kohberger R. Serum serotype-specific pneumococcal anticapsular immunoglobulin G concentrations after immunization with a 9-valent conjugate pneumococcal vaccine correlate with nasopharyngeal acquisition of pneumococcus. J Infect Dis 2005;192(3):367-76.

[34] Dagan R, Juergens C, Trammel J, Patterson S, Greenberg D, Givon-Lavi N, et al Modeling pneumococcal nasopharyngeal acquisition as a function of anticapsular serum antibody concentrations after pneumococcal conjugate vaccine administration. Vaccine 2016;34(36):4313-20.

[35] Fleming-Dutra KE, Conklin L, Loo JD, Knoll MD, Park DE, Kirk J, et al. Systematic review of the effect of pneumococcal conjugate vaccine dosing schedules on vaccine-type nasopharyngeal carriage. Pediatr Infect Dis J 2014;33 Suppl 2: S152-60.

[36] Pennington SH, Pojar S, Mitsi E, Gritzfeld JF, Nikolaou E, Solorzano C, et al. Polysaccharide-specific memory $B$ cells predict protection against experimental human pneumococcal carriage. Am J Respir Crit Care Med 2016;194(12):1523-31. 